\title{
IMPACTOS DEL CAMBIO CLIMÁTICO EN LA ESTACIONALIDAD TURÍSTICA
}

\author{
Domingo F. RASILLA ÁLVAREZ1', Guillermo CALLEJA HERRERO \\ ${ }^{1}$ Depto. de Geografia, Urbanismo y OT. Universidad de Cantabria. \\ domingo.rasilla@unican.es
}

\section{RESUMEN}

Este trabajo analiza la relación entre el clima y la demanda turística en diferentes destinos turísticos internacionales y explora el impacto de dos escenarios de cambio climático en la estacionalidad de la actividad turística. Los resultados muestran que la relación entre clima y demanda turística muestra sensibles diferencias entre destinos, a causa de las distintas ofertas turísticas y mercados que los utilizan, siendo los situados en latitudes medias los que ofrecen una relación más clara. Las proyecciones mejoran la aptitud turística general a través de sustanciales cambios en la estacionalidad de la demanda turística.

Palabras clave: Clima, TCI, Turismo, Cambio climático.

\begin{abstract}
This memory analyzes the relationship between climate and tourism demand in several international tourist destinations and explores the impact of two climate change scenarios on tourism demands.

The results show that the relationship between climate and tourism demand differs among destinations because of the different tourism attractions and markets, the Mediterranean region being which displays a closest relationship. Tourism demand projections show an overall improvement of the suitability, through substantial changes in tourism demand seasonality.
\end{abstract}

Key words: Climate, TCI, Tourism, Climate Change.

\section{INTRODUCCIÓN}

El turismo es uno de los sectores económicos más dinámicos a escala mundial, habiendo experimentado un fuerte crecimiento a lo largo del último tercio del siglo XX; actualmente, su aportación al PIB mundial es superior al 10\% (WTO et al., 2013). Para algunos países constituye el principal motor económico, y en muchos otros es clave para compensar los déficits en la balanza comercial, caso de Grecia o España, donde los ingresos por turismo suponen un 15\% del PIB nacional, frente al 5,5\% de la década de los 70 (Prados et al., 2003). Aunque en la actualidad prácticamente la totalidad de los países actúan indistintamente como emisores o receptores de turistas, el flujo internacional de viajeros sigue estando dominado por los desplazamientos desde latitudes medias a las subtropicales e intertropicales, constituyendo el clima uno de los factores que mayor poder de atracción ejercen sobre los turistas (Amelung et al., 2007; Gómez Martín, 2005; Hamilton et al., 2005). 
Sin embargo, determinar el impacto del tiempo y del clima sobre la actividad turística no es una tarea sencilla, dado que no todos los turistas tienen las mismas preferencias, y la propia actividad turística engloba diferentes opciones que requieren condiciones atmosféricas diversas. Una dificultad añadida es cuantificar el papel de cada variable meteorológica sobre la práctica turística, evaluando de manera objetiva la idoneidad de un destino. Con este propósito, la aplicación inicial de índices bioclimáticos clásicos fue complementada por otros desarrollados específicamente para el turismo, como el "Tourism climate index" de Mieczkowski (1985), su modificación por Morgan (2000), el "Beach climate index" (Becker, 1998) y el "Climate tourism index" (de Freitas, 2008). Su validación requiere su contraste con datos de demanda turística, habitualmente difíciles de conseguir (Scott et al., 2004; Amelung y Viner, 2006).

Crecientes evidencias indican que el clima terrestre está experimentado una serie de cambios en comparación con la época preindustrial, y estos cambios se acentuarán a lo largo del s. XXI. En su último informe, el IPCC considera que es muy probable que la evolución del sistema climático se acelere si prosiguen las emisiones de gases de efecto invernadero al ritmo actual, con una estimación de aumento de las temperaturas de entre 1,8 y $4^{\circ} \mathrm{C}$. Este calentamiento vendrá acompañado, igualmente, de cambios en la magnitud, distribución espacial y régimen de otros parámetros climáticos, como precipitación, nubosidad o viento etc...

Es evidente, por tanto, la necesidad de afrontar sus consecuencias a través de la adaptación y mitigación (Wilbanks et al. 2007). Los impactos estimados del cambio climático en las actividades turísticas se pueden agrupar en dos grandes categorías. Por un lado, los directos, en relación a su capacidad para determinar la localización de los destinos, la gama de actividades turísticas, la estacionalidad de la demanda y los costos de operación (calefacción/refrigeración, suministro de agua y alimentos, coste de los seguros etc...). Por otro lado, si la frecuencia o magnitud de ciertos extremos climáticos (olas de calor, sequías, huracanes) también varía, la industria turística se verá afectada a través del coste adicional para la reparación de daños o unos gastos de explotación más elevados, consecuencia de la necesidad de asegurar los bienes en situación de riesgo. Cabe señalar por último, que el cambio climático actuará también sobre otros recursos, como la disponibilidad de agua, la biodiversidad, el paisaje, el nivel del mar etc..., acentuando la pérdida de competitividad de algunos destinos.

En relación a lo expuesto, los objetivos de este trabajo son dos:

1. Analizar la relación entre clima y demanda turística en varios destinos turísticos internacionales, comparando los valores del índice TCI con indicadores de demanda turística.

2. Explorar el impacto que el cambio climático podrá causar en la estacionalidad de la demanda turística en los citados destinos.

\section{FUENTES DE INFORMACIÓN Y METODOLOGÍA}

En relación con los objetivos, se seleccionaron 10 destinos turísticos, siguiendo como criterios su relevancia internacional, su diversidad climática y la disponibilidad de información accesible a través de Internet. La mayoría pertenecen a destinos de sol y playa, pero se añadieron dos destinos de naturaleza en los que predominan activida- 
des al aire libre (trekking, senderismo etc...). La demanda turística está representada por los promedios mensuales, correspondientes al menos a 5 años completos, del número de visitantes o de pasajeros de los aeropuertos más próximos. Los datos brutos mensuales fueron transformados en porcentajes con respecto al promedio anual para el cálculo de la estacionalidad de la demanda.

Los datos climáticos, correspondientes a la estación más cercana al destino turístico seleccionado fueron extraídos mayoritariamente de la herramienta Climate Change World Weather File Generator for World-Wide Weather Data- CCWorldWeatherGen (http://www.energy.soton.ac.uk/ccworldweathergen/). Esta herramienta proporciona valores medios mensuales de temperatura, precipitación, insolación, humedad relativa y viento, para el periodo 1971-2000, y también genera proyecciones de esas mismas variables para el periodo 2071-2100, basadas en el modelo HadCM3. Para este trabajo se han seleccionado los escenarios A2 y B2. El primero, basado en un modelo de desarrollo agresivo, reproduce un fuerte crecimiento demográfico y económico, con ausencia de políticas e innovaciones tecnológicas en beneficio del medio ambiente. El escenario B2 presenta un importante crecimiento de la población y de la economía, pero a un ritmo más lento, y una mayor preocupación por el medio ambiente, que se traduce en políticas para combatir al cambio climático y en la búsqueda de nuevas tecnologías para reducir la dependencia de los combustibles fósiles.

El TCI (Tourism Climate Index, Mieczkowski, 1985) es uno de los índices de aptitud climático-turística más utilizados al integrar las tres facetas climáticas esenciales (térmicas, estéticas y físicas), combinando los promedios mensuales de 7 variables meteorológicas, según una fórmula que incorpora factores de ponderación para resaltar la importancia relativa de cada una de ella:

$$
\mathrm{TCI}=(8 * \mathrm{CLD})+(2 * \mathrm{CLA})+(4 * \mathrm{R})+(4 * \mathrm{~S})+(2 * \mathrm{~W})
$$

\begin{tabular}{|c|c|c|c|c|}
\hline Componente & Subíndice & Variable & Descripción & $\begin{array}{c}\text { Ponderación } \\
(\%)\end{array}$ \\
\hline CID & Confort diurno & $\begin{array}{c}\text { Temperatura máxima diaria } \\
\text { y humedad relativa mínima }\end{array}$ & $\begin{array}{c}\text { Confort térmico en } \\
\text { las horas de máxima } \\
\text { actividad }\end{array}$ & 40 \\
\hline CIA & Confort diario & Temperatura y humedad relativa & Confort térmico diurno & 10 \\
\hline P & Precipitación & Promedio mensual & Negativo & 20 \\
\hline S & Insolación & Horas de sol & Positivo & 20 \\
\hline W & Viento & Promedio & Desigual & 10 \\
\hline
\end{tabular}

Cuadro I. Componentes del índice TCI

Se considera que un valor superior a 70 es demostrativo de una buena aptitud turística; por el contrario, meses por debajo de 40 indican una pobre aptitud (Amelung y Viner, 2006).

La relación entre la demanda y la aptitud turística se evaluó mediante un modelo de regresión lineal entre los porcentajes mensuales de demanda turística (variable dependiente) y los valores mensuales de aptitud climático- turística (variable independiente o predictora), para desarrollar a continuación una ecuación que será utilizada con fines predictivos. 


\section{RESULTADOS}

\subsection{Relación entre aptitud climático-turística y demanda turística.}

El cuadro II muestra el potencial climático turístico actual en los destinos seleccionados. Los valores inferiores a 40 aparecen resaltados en color verde, mientras que los valores superiores a 70 aparecen en rojo, con el fin de facilitar la comparación entre destinos.

\begin{tabular}{|l|c|c|c|c|c|c|c|c|c|c|c|c|c|}
\hline & ENE & FEB & MAR & ABR & MAY & JUN & JUL & AGO & SEP & OCT & NOV & DIC & ANUAL \\
\hline Denali & 8 & 14 & 22 & 31 & 45 & 62 & 73 & 66 & 53 & 33 & 20 & 12 & 37 \\
\hline Yellowstone & 41 & 46 & 55 & 61 & 74 & 89 & 92 & 94 & 88 & 66 & 50 & 41 & 66 \\
\hline Reus & 58 & 60 & 62 & 68 & 78 & 93 & 95 & 89 & 84 & 75 & 61 & 58 & 73 \\
\hline Tenerife & 80 & 82 & 86 & 86 & 90 & 96 & 98 & 97 & 94 & 92 & 87 & 82 & 89 \\
\hline Honolulu & 84 & 86 & 86 & 90 & 92 & 92 & 91 & 90 & 89 & 86 & 84 & 82 & 88 \\
\hline Arica & 98 & 98 & 97 & 89 & 79 & 70 & 65 & 63 & 67 & 75 & 84 & 93 & 82 \\
\hline Camboya & 80 & 82 & 78 & 75 & 73 & 66 & 68 & 65 & 62 & 60 & 70 & 74 & 71 \\
\hline San José & 90 & 89 & 90 & 81 & 67 & 65 & 71 & 67 & 64 & 64 & 72 & 86 & 76 \\
\hline Tahiti & 62 & 62 & 63 & 66 & 74 & 79 & 78 & 78 & 75 & 76 & 68 & 61 & 70 \\
\hline Seychelles & 61 & 67 & 66 & 65 & 70 & 74 & 74 & 74 & 71 & 72 & 68 & 64 & 69 \\
\hline
\end{tabular}

Cuadro II. Valores medios mensuales del TCI según destino turístico.

Aplicando los criterios propuestos por Scott y McBoyle (2004), los destinos seleccionados pertenecen a 4 modelos de distribución estacional; no están presentes ni el régimen desfavorable (típico de ámbitos árticos) ni el bimodal, característico de regiones áridas.

El régimen con máximo estival está representado por los destinos de latitudes medias y altas, donde el verano es el periodo más favorable, aunque los contrastes son evidentes en función del tipo de clima (en particular del régimen térmico). Así, mientras en los ámbitos mediterráneos (Reus) el contraste estacional es reducido (entre 58 y 95 puntos, en torno a 6 meses óptimos y sin ningún mes por debajo de 40 puntos), en ámbitos subárticos (Denali) resalta un invierno largo y poco favorable ( 7 meses por debajo de 40 puntos) y un corto verano (sólo julio supera los 70 puntos). El clima continental de Yellowstone se sitúa a medio camino, con un periodo desfavorable más corto ( 3 meses) y uno favorable más prolongado (5 meses). La correspondencia entre aptitud y demanda turística es considerable: el 75\% de los visitantes se desplazan en verano, frente a menos del $5 \%$ en invierno. La influencia del clima debe relativizarse a favor de factores como la proximidad a los centros emisores de visitantes, nacionales en el caso de los parques de EEUU o comunitarios en el de Reus, al igual que su perfil socioeconómico; en el caso de los parques naturales de EEUU suelen ser amantes de las actividades al aire libre (trekking, senderismo, etc...; http://www. nps.gov/dena/planyourvisit/things2do.htm ), mientras que Reus ha sido el paraíso del turismo "low cost" con estancias breves buscando sol, playa y ocio nocturno, a veces complementado con visitas a parques temáticos.

Dos destinos turístico (Canarias y Hawai) reproducen el conocido como régimen óptimo todo el año, pues ningún mes posee un TCI $\leq 80$, lo que se traduce en la ausencia de estacionalidad (aunque el verano muestra valores ligeramente superiores). En 
comparación con los valores de demanda turística, se observa una relación moderada, pero negativa, dado que la época con más visitantes es el invierno.

Los destinos San José de Costa Rica y Phnom Penh muestran un régimen con un "máximo invernal", típico de latitudes intertropicales. En estos lugares, el solsticio de invierno es más confortable, debido a temperaturas frescas, alisios constantes y sobre todo, a un volumen de precipitaciones mucho menor que en el solsticio de verano. La relación entre aptitud turística y demanda es significativa y de nuevo positiva; incluso, se percibe en San José un ligero aumento de visitantes coincidiendo con un periodo de remisión de las precipitaciones conocido popularmente como "veranillo". En Phnom Penh también se aprecia este pico secundario en julio y agosto, en pleno monzón de verano; dado que no existe una remisión de las precipitaciones análoga al de San José, este segundo máximo se puede atribuir al periodo de vacaciones habitual en los países desarrollados del hemisferio norte, dado que en ambos casos, la mayoría de los turistas proceden de América del Norte o Europa, buscando un tipo de esparcimiento basado también en turismo natural y de aventura (Costa Rica cuenta con algunas de las mejores reservas biológicas del planeta) y el cultural (Khemer). Por su parte, otro destino que disfruta de un clima muy atractivo para el turismo es Arica, dada su moderación térmica y ausencia de precipitaciones, aunque la frecuencia de nieblas costeras ("camanchaca") durante el solsticio de invierno explicaría la reducción del número de visitantes durante este periodo del año.

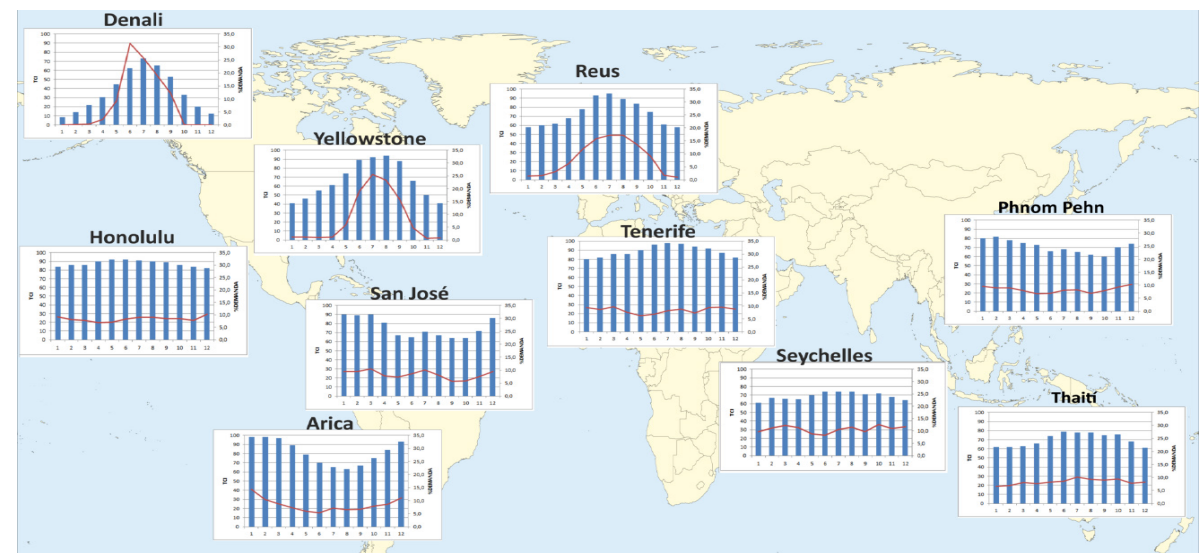

Figura 1. Régimen anual del TCI (barras) y la demanda turística (líneas) correspondiente a los destinos seleccionados.

Los observatorios de Tahití y Male (Seychelles) corresponden al tipo con "máxima aptitud durante la estación seca". Por su localización cercana a la ZCIT y reducida variabilidad térmica, la aptitud viene determinada por el régimen de precipitaciones, con máximos durante el alisio seco del SE, que coincide con el periodo de vacaciones en el Hemisferio N, siendo el principal mercado turístico personas con alto poder adquisitivo, de nacionalidad europea, estadounidense y recientemente, rusa o china. Sin embargo, cabe destacar la ausencia de relación entre aptitud turística y demanda en Seychelles. Sin descartar errores en los datos de demanda consultados (basados en una muestra de 5 años, que podría magnificar variaciones puramente aleatorias), una explicación alternativa es una oferta basada en un amplio abanico de actividades, como los deportes 
acuáticos (vela, submarinismo, windsurf, surf...), el turismo de naturaleza (gran riqueza biológica tanto marina como terrestre) etc., menos condicionado por el clima.

\subsection{Cambios en la aptitud turística y la estacionalidad de la demanda causados por el cambio climático.}

De la aplicación las relaciones estadísticas encontradas entre aptitud turística y demanda, bajo las condiciones actuales, a los escenarios de cambio climático propuestos, se deduce que, para finales del siglo XXI, la aptitud turística aumentará en destinos ubicados en latitudes medias y altas, disminuyendo en los situados en latitudes bajas. Para analizar con detenimiento estos cambios, se hará hincapié en las variaciones en la aptitud turística (TCI anual) y en la longitud de la estación óptima (número de meses con TCI>70; cuadro III). La figura 2 ofrece una visión más amplia de los cambios esperados.

\begin{tabular}{|l|c|c|c|c|c|c|l|}
\hline & TCI & & \multicolumn{4}{|c|}{ Num meses TCI > 70 } & \\
\hline & ACTUAL & B2 & A2 & ACTUAL & B2 & A2 & Resultado \\
\hline Denali & 37 & 44 & 47 & 1 & 2 & 3 & Aumento \\
\hline Yellowstone & 66 & 70 & 71 & 5 & 6 & 7 & Aumento \\
\hline Reus & 73 & 75 & 75 & 6 & 7 & 8 & Aumento \\
\hline Tenerife & 89 & 93 & 94 & 12 & 12 & 12 & Igual \\
\hline Honolulu & 88 & 85 & 79 & 12 & 12 & 10 & Disminución \\
\hline Arica & 82 & 88 & 90 & 8 & 12 & 12 & Aumento \\
\hline Camboya & 71 & 69 & 63 & 6 & 6 & 3 & Disminución \\
\hline San José & 76 & 74 & 69 & 7 & 6 & 4 & Disminución \\
\hline Tahiti & 70 & 61 & 54 & 6 & 3 & 1 & Disminución \\
\hline Seychelles & 69 & 60 & 53 & 5 & 1 & 0 & Disminución \\
\hline
\end{tabular}

Cuadro III. Cambios en el valor anual del TCI para el periodo 2070-2100 bajo los escenarios A2 y $B 2$.

En los observatorios de latitudes medias y altas la mejora de la aptitud turística general estará condicionada fundamentalmente por el impacto de la elevación de las temperaturas. Por ejemplo, la mejora en Denali provendrá de la reducción del frío invernal y la prolongación del periodo estival, aunque mitigado por un aumento simultáneo de las precipitaciones. El régimen de aptitud turística mantendrá su máximo de verano, alargando la duración del periodo óptimo hasta 3 meses. En Yellowstone, el aumento de las temperaturas alargará la estación óptima (ahora 7) pero exacerbará el carácter continental del verano, lo cual hace esperar una cierta reducción de la concentración estival de la demanda a favor de las estaciones equinocciales (mayo y septiembre-octubre) y una consecuente transformación hacia un régimen bimodal, particularmente en el escenario A2. Una evolución análoga es esperable en los destinos mediterráneos (Reus), donde el considerable aumento térmico y la disminución de las precipitaciones, sobre todo primaverales, reducirán la aptitud estival, pero mejorará la primaveral (marzo, abril y mayo), y en menor medida de la otoñal (septiembre y 
octubre). La demanda responderá en la misma dirección, desplazándose hacia la primavera, como ya ha sido propuesto en estudios anteriores (Amelung y Viner, 2006).

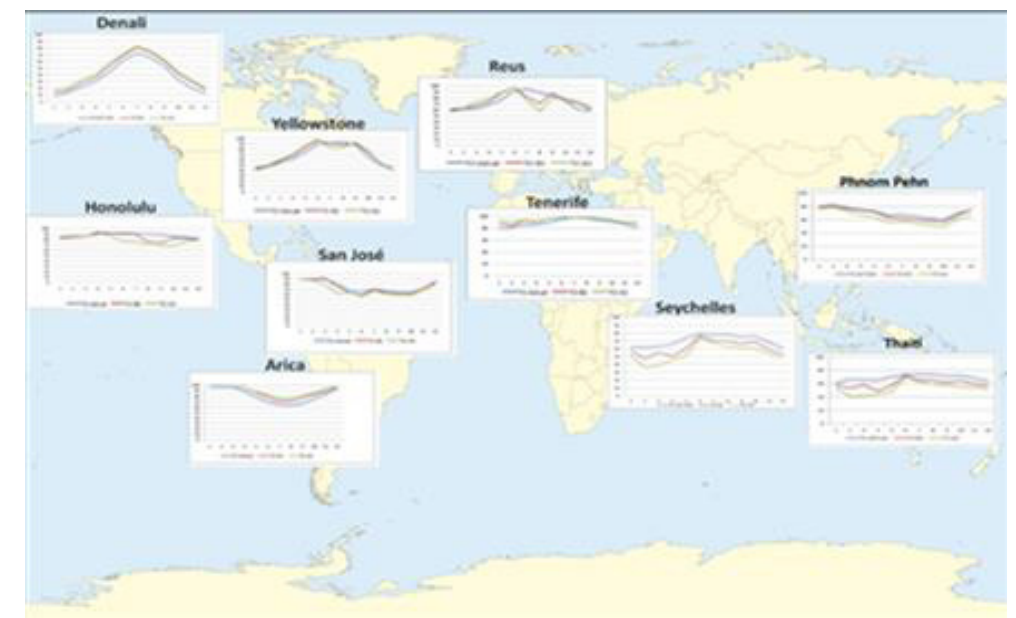

Figura 2. Cambios esperados en el régimen anual del TCI para el periodo 2070-2100 bajo los escenarios $A 2$ y $B 2$.

En las localizaciones con un régimen óptimo no se producirá una mejora adicional a su ya elevada aptitud turística. Tanto en Canarias como Hawai los cambios proyectados igualarán la aptitud turística de los meses de invierno y primavera a los de verano. En el caso de Canarias, dada la escasez de precipitaciones, la causa fundamental de la mejora invernal son las temperaturas, que en verano están amortiguadas por la corriente fría de las Canarias. El impacto de estos cambios climáticos sobre la demanda turística tenderá hacia una homogenización estacional, con valores similares a lo largo de todo el año. De hecho, y siempre en función de los datos aquí registrados, Canarias podría beneficiarse si, manteniendo su oferta invernal, los flujos estivales europeos prefieren otros destinos ante el rigor térmico de los ámbitos mediterráneos. Por su parte, Honolulu experimentará un marcado decrecimiento de su aptitud turística (reducción del valor anual de 88 a 79, sólo 10 meses aptos), particularmente durante el verano (julio y agosto), ya que no disfruta del efecto amortiguador de una corriente fría. La mejora de la aptitud turística invernal no sería, por tanto, suficiente para compensar la tendencia general, por lo que mantendrá un régimen óptimo todo el año.

Dado que el aumento térmico ligado al calentamiento global será más limitado en los trópicos, son los cambios en la precipitación y/o insolación, los que explican las modificaciones en la aptitud turística. En los destinos con un máximo invernal se espera un cierto empeoramiento de los niveles generales de aptitud y de la longitud del periodo óptimo anual, al coincidir dos procesos desfavorables, el aumento notable de las temperaturas durante la estación seca y el exceso de las precipitaciones durante la húmeda, algo más acentuado en Pnhom Pehn. Las proyecciones de demanda ofrecen una imagen relativamente compleja, con ligeras variaciones de escasa entidad, tendiendo a reducir la estacionalidad de su demanda. A diferencia de los otros dos destinos subtropicales, Arica experimentará una sustancial mejora de su aptitud turística (el 
promedio anual del TCI pasará de 82 a 90; el número de meses óptimos a 12), debido fundamentalmente a que el calentamiento global se traducirá en una menor probabilidad de nieblas costeras durante el invierno, mientras que la corriente fría de Humboldt seguirá moderando las temperaturas estivales. Como consecuencia, también es esperable una homogeneización de la demanda a lo largo del año.

Sin embargo, serán los destinos más lluviosos y con régimen de estación seca los que experimenten un mayor retroceso. Así, Tahití sufrirá un fuerte retroceso de la aptitud turística, con una caída de 6 puntos en su TCI y una reducción del número de meses favorables desde 6 hasta 1. Este empeoramiento se debe principalmente al efecto combinado de aumento de la temperatura y de las precipitaciones. Como consecuencia de ello, la estacionalidad de la demanda se acentuará, con una concentración adicional durante la estación seca. En el caso de Seychelles, al no existir una relación significativa entre aptitud y demanda no se ha realizado ninguna proyección.

\section{DISCUSIÓN Y CONCLUSIONES}

El objetivo de este trabajo ha sido identificar y cuantificar la relación entre la aptitud climático-turística y el régimen anual de demanda turística, y posteriormente, determinar cómo afectará el cambio climático a dicha demanda turística. Para ello se seleccionaron una serie de destinos turísticos de relevancia mundial, calculándose el valor mensual del índice turístico de Mieczkowski (TCI). Estos valores fueron comparados con datos de demanda turística a través de la elaboración de un simple modelo estadístico, utilizado posteriormente para obtener proyecciones de demanda futura bajo dos escenarios de cambio climático.

En relación al primer aspecto, cabe señalar que la relación entre demanda turística y condiciones climáticas no siempre es significativa y ni siquiera positiva, llegando a invertirse en algún destino por el comportamiento de los principales mercados a los que dirige su oferta. La relación más estrecha, de carácter positivo, es característica de latitudes medias y altas, que muestran un máximo estival de actividad (predominio de turistas de clase media). La relación es positiva, pero más débil, en la mayoría de los ámbitos tropicales y monzónicos ligados a una marcada estacionalidad climática. La causa de esta menor conexión estaría vinculada a alguna de las conocidas deficiencias del TCI, que al estar basado en promedios mensuales de precipitación, podría sobreestimar el efecto negativo de las precipitaciones convectivas. Merece especial atención la relación negativa entre aptitud turística y demanda en destinos subtropicales bajo la influencia de los alisios (Canarias y Hawai). Amén de una notable estabilidad en las condiciones atmosféricas a lo largo de todo el año, ambas localizaciones son destinos refugio para mercados europeos y americanos durante el periodo de rigor invernal; su atractivo disminuye durante la estación cálida, por la competencia de destinos clásicos, como el Mediterráneo, más próximos y baratos a los mercados domésticos europeo y estadounidense.

En cuanto al impacto previsible del cambio climático en la demanda turística, el análisis señala impactos significativos tanto en la aptitud general como en la duración y estacionalidad de los periodos favorables para el desarrollo de actividades turísticas. En relación al primer aspecto, se aprecia un patrón espacial claro: mejora en latitudes medias y altas (Denali, Yellowstone y Reus), estabilidad en climas secos 
subtropicales (aumento en Arica, mantenimiento en Tenerife, reducción en Honolulu) y disminución en climas húmedos tropicales, ya que al aumento térmico durante la estación seca se añadirá el aumento de las precipitaciones durante la estación húmeda. En los subtrópicos la diversidad podría estar relacionada con las incertidumbres asociadas al aumento de las temperaturas en destinos próximos a afloramientos de corrientes frías. Las mejorías previsibles en latitudes medias y altas están acompañadas de una prolongación de la estación favorable a la actividad turística, sin cambio de régimen ("máximos estivales"), o bien de una redistribución de los meses más favorables a la práctica turística, lo que supone un cambio de régimen de "máximos estivales" a "máximos equinocciales". Esta última circunstancia afectará a destinos turísticos como el Mediterráneo, obligando a un rediseño de la oferta turística en un intento de compensar los flujos turísticos hacia destinos más septentrionales.

Cabe concluir que, aunque este y otros trabajos similares constituyen una primera aproximación a los impactos del cambio climático, sus resultados son claramente mejorables. En primer lugar, el clima no es el único factor que modula la actividad turística a escala global, sobre todo a gran escala, En realidad, existe una amalgama de factores implicados, entre los que se incluyen los económicos (ingresos de los turistas, precios de los destinos, costo del desplazamiento), los ambientales (paisajes) o incluso sociales (herencia cultural). Más aún, no sólo las condiciones climáticas del destino son importantes, sino también las de las áreas emisoras (Hamilton et al., 2005). Como se ha puesto de manifiesto de manera repetida en los últimos años, la estabilidad política y/o la situación económica condicionan de manera sustancial el potencial turístico de un país. En segundo lugar, este trabajo aborda el problema desde una óptica general, requiriéndose estudios específicos para las distintas modalidades turísticas, cuyas preferencias climáticas son aún desconocidas. Además, cabe señalar la disparidad en el formato (espacial y temporal) de la información climática y turística, ya que normalmente es difícil encontrar datos de demanda segregados con una resolución temporal inferior al año y desagregados a escala sub-nacional. Finalmente, todavía persisten numerosas incertidumbres en las proyecciones de cambio climático, dado que éstas resultan de unos escenarios teóricos de desarrollo económico y social y de unos modelos climáticos en continuo proceso de mejora.

\section{AGRADECIMIENTOS}

Este trabajo está basado en el Trabajo de Fin de Grado Impactos del cambio climático en la estacionalidad turística, elaborado para la obtención del Grado en Geografía y Ordenación del Territorio. Fue leído el 4 de septiembre de 2015 en la Facultad de Filosofía y Letras de la Universidad de Cantabria, siendo su autor Guillermo Calleja Herrero.

\section{REFERENCIAS}

Amelung, B y Viner, D. (2006). "Mediterranean tourism: exploring the future with the tourism climatic index". Journal of Sustainable Tourism, 14, pp. 349-366.

Amelung, B; Nicholls, S y Viner, D. (2007). "Implications of global climate change for tourism flows and seasonality". Journal of Travel Research, 45, pp. 285-296. 
Becker, S. (1998). "Beach Comfort Index-A new approach to evaluate the thermal conditions of beach holiday resorts using a South African example". GeoJournal, 44, pp. 297-307.

De Freitas, C. (2003). "Tourism climatology: evaluating environmental information for decision making and business planning in the recreation and tourism sector". International Journal of Biometeorology, 48, pp. 45-54.

Gómez Martín, B (2005). "Reflexión geográfica en torno al binomio clima-turismo". Boletín de la Asociación de Geógrafos Españoles, 40, pp. 111-134.

Hamilton, J. (2005). Climate and the Destination Choice of German Tourists. Centre for Marine and Climate Research, Hamburg University, Hamburg, Germany. http://www.fnu.zmaw.de/fileadmin/fnu-files/models-data/htm/climtour.pdf [Consulta: 1 de septiembre de 2015].

Hamilton, JM. y Lau, MA. (2005). "The role of climate information in tourist destination choice decision-making". En: Gossling S. y Hall CM. (Eds). Tourism and Global Environmental Change. London: Routledge.

Mieczkowski, Z. (1985). "The Tourism Climatic Index: A method of evaluating world climates for tourism". Canadian Geographer, 29, pp. 220-233.

Morgan, R.; Gatell, E.; Junyent, R.; Micallef, A.; Ozhan, E. y Williams, A. (2000). "An improved user-based Beach Climate Index". Journal of Coastal Conservation, 6, pp. 41-50.

Prados, L (2003). “El progreso económico de España (1850-2000)". Las estimaciones del Consejo de Economía Nacional, pp. 32: 34.

Scott, D; McBoyle, G y Schwartzentruber, M. (2004). "Climate change and the distribution of climatic resources for tourism in North America". Climate Research, 27, p. 105-117.

UNWTO (2013). UNWTO world tourism barometer. On-Line: http://dtxtq4w60xqpw. cloudfront.net/sites/all/files/pdf/unwto_barom13_02_apr_excerpt.pdf [Consulta: 1 de septiembre de 2015].

Wilbanks, TJ. et al. (2007). "Industry, Settlement and Society". En: Parry ML et al. (eds.): Climate Change 2007: Impacts, Adaptation and Vulnerability. Contribution of Working Group II to the Fourth Assessment Report of the IPCC, Cambridge University Press, Cambridge and New York, p. 357-390. 